\title{
Noise autocorrelation spectroscopy with coherent Raman scattering
}

\author{
XIAOJI G. XU, STANISLAV 0. KONOROV, JOHN W. HEPBURN AND VALERY MILNER* \\ Department of Chemistry and the Laboratory for Advanced Spectroscopy and Imaging Research (LASIR), University of British Columbia, 2036 Main Mall, Vancouver, BC, \\ V6T 1Z1, Canada \\ *e-mail: vmilner@chem.ubc.ca
}

Coherent anti-Stokes Raman scattering (CARS) with femtosecond laser pulses has become a widespread method in nonlinear optical spectroscopy and microscopy ${ }^{1,2}$. As a third-order nonlinear process, femtosecond CARS exhibits high efficiency at low average laser power. High sensitivity to molecular structure enables detection of small quantities of complex molecules ${ }^{3,4}$ and non-invasive biological imaging ${ }^{5}$. Temporal and spectral resolution of CARS is typically limited by the duration of the excitation pulses and their frequency bandwidth, respectively. Broadband femtosecond pulses are advantageous for time-resolved CARS spectroscopy ${ }^{6,7}$, but offer poor spectral resolution. The latter can be improved by invoking optical ${ }^{8,9}$ or quantum ${ }^{10,11}$ interference at the expense of increasing complexity of instrumentation and susceptibility to noise. Here, we present a new approach to coherent Raman spectroscopy in which high resolution is achieved by means of deliberately introduced noise. The proposed method combines the efficiency of a coherent process with the robustness of incoherent light. It does require averaging over different noise realizations, but no temporal scanning or spectral pulse shaping as commonly used by frequency-resolved spectroscopic methods with ultrashort pulses.

In coherent anti-Stokes Raman scattering (CARS), pump and Stokes photons of frequencies $\omega_{\mathrm{p}}$ and $\omega_{\mathrm{S}}$, respectively, excite molecular vibrations at frequency $\Omega=\omega_{\mathrm{p}}-\omega_{\mathrm{s}}$ (see Fig. 1a). A probe photon at $\omega_{0}$ is scattered off the coherent vibrations generating the anti-Stokes signal at frequency $\omega=\left(\omega_{0}+\Omega\right)$. Special care is usually taken to preserve the coherence of laser pulses as it determines the accuracy of a spectroscopic measurement. The latter can be improved by means of the pulse-shaping technique ${ }^{12,13}$, which modifies the amplitudes and phases of the spectral constituents of the pulse ${ }^{14}$ (see Fig. $1 \mathrm{~b}(\mathrm{i})$ ). It enables selective excitation ${ }^{10,11}$ or selective probing ${ }^{8,9}$ of separate vibrational modes on the frequency scale narrower than the overall pulse bandwidth. Selective excitation is based on the coherent control ${ }^{15}$ of nonlinear optical processes ${ }^{16}$, whereas selective probing uses the idea of multiplex CARS 5 in which a small part of the pulse spectrum is modified (in amplitude, phase or polarization) and serves as a narrowband probe. Both approaches rely on the effects of quantum or optical interference, and are therefore sensitive to noise and decoherence, which often ruin the spectral and temporal integrity of the pulse. Using a narrow slice of the available pulse spectrum as a probe in multiplex CARS also means that higher resolution may only be obtained at the expense of the proportionally lower probe power and correspondingly lower signal.
Here, we show that contrary to the common belief that spectral noise is detrimental to coherent spectroscopic measurements, it can be used for improving the resolution, efficiency and robustness against unavoidable degradation of pulse coherence. In our new method of noise autocorrelation spectroscopy with coherent antiStokes Raman scattering (NASCARS), the laser-induced molecular vibrations are probed by a broadband pulse with intentionally randomized amplitude and phase. The vibrational resonances result in and are identified through the appearance of intensity correlations in the noisy spectrum of coherently scattered photons. Owing to the presence of Raman resonances, the spectral noise of the probe field is transferred to the spectrum of the scattered photons. Although completely uncorrelated in the probe, the noise in the anti-Stokes field acquires strong correlations as a result of the molecular vibrational coherence. The latter assures that multiple Raman modes 'map' the same realization of the probe noise onto the different spectral regions of the generated anti-Stokes spectrum, as shown schematically in Fig. 1a. An autocorrelation of the noisy anti-Stokes spectrum corresponds directly to the autocorrelation of the Raman mode structure, thus revealing the vibrational beating frequencies of the medium. Even though the probe in NASCARS carries the full available spectral bandwidth of a femtosecond pulse, the resolution is determined by the much smaller frequency correlation length of the introduced noise, $\delta \omega_{n}$. Use of the full bandwidth provides the ability to increase the resolution with no penalty in the available probe power and, therefore, with no direct reduction of the signal level. By its very nature, the method is not sensitive to the quality of the temporal and spectral profile of the pulses, and can be implemented by simply destroying the coherence of a laser pulse, as demonstrated here.

Noise and fluctuations in resonant fluorescence ${ }^{17}$, scattered light ${ }^{18}$ and number of trapped ultracold atoms ${ }^{19}$ have been used to observe statistical properties of a medium but did not provide any spectroscopic information. NASCARS extends the idea of using noise correlations as a diagnostic tool to the domain of coherent nonlinear spectroscopy with ultrashort pulses. Noisy nanosecond lasers have previously been exploited in coherent Raman spectroscopy as a means of achieving time resolution shorter than the duration of the excitation pulses ${ }^{20,21}$. The method of coherence observation by interference noise ${ }^{22}$ has been introduced for detecting the decay rates of electronic coherence, and applied to linear spectroscopy with narrowband picosecond pulses and to quantum state holography ${ }^{23}$. Unlike NASCARS, both approaches retrieve spectroscopic data from scanning the time delay between the noisy pulses. Here, we show that the availability 
a

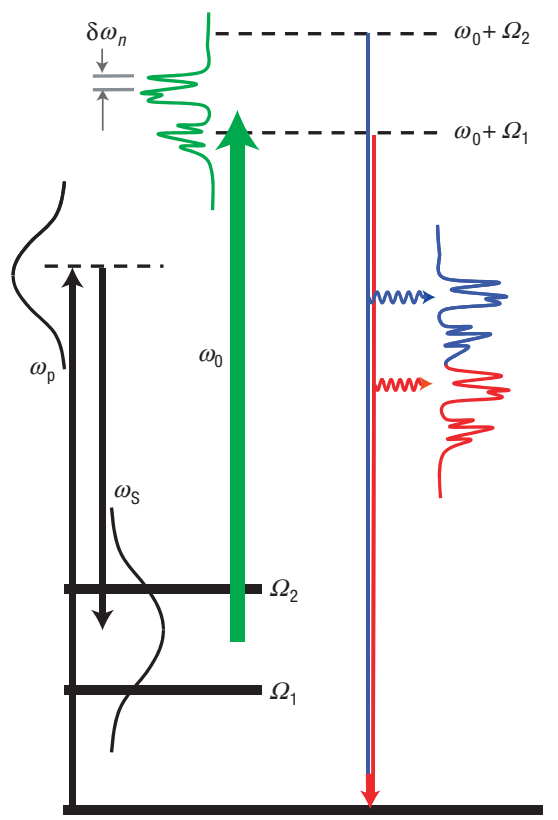

b

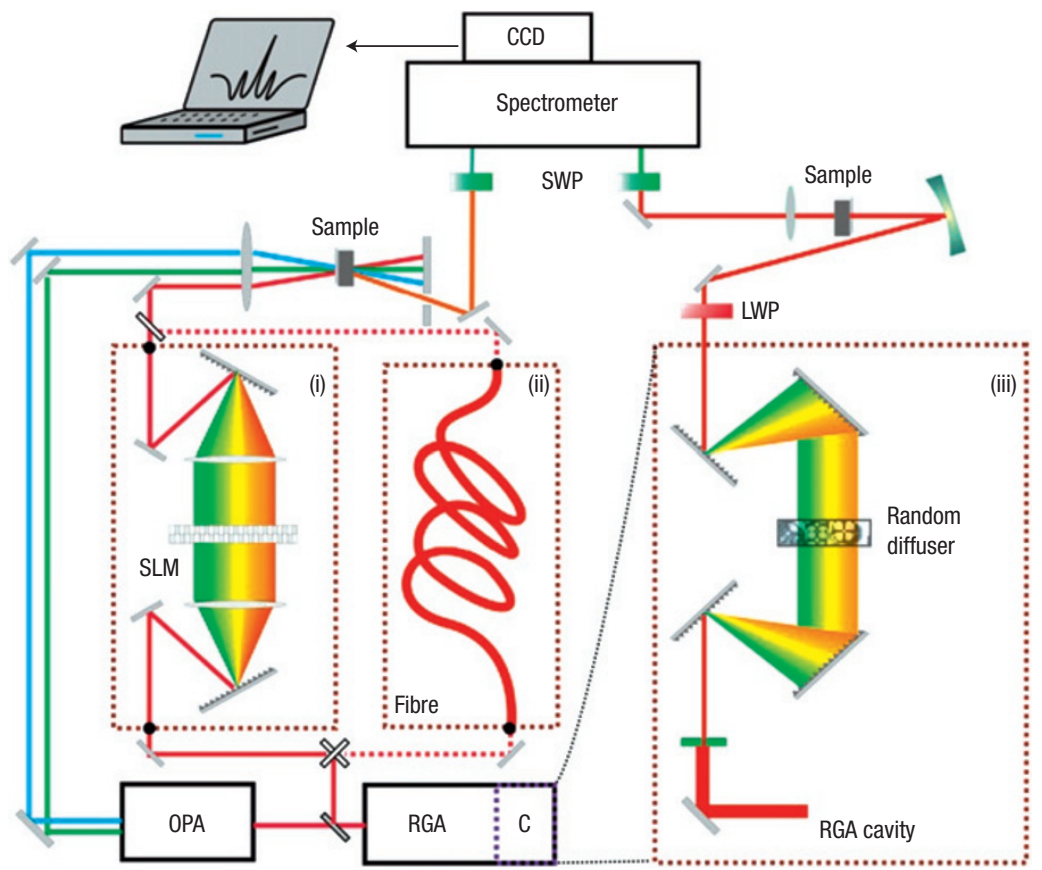

Figure 1 Interaction scheme for CARS and experimental arrangement for the detection of NASCARS signal. a, Pump and Stokes pulses (black) overlap in time and induce the coherent molecular vibrations. The broadband probe pulse (green) interacting with the vibrational energy levels $\Omega_{1,2}$ generates the anti-Stokes light (red and blue). In NASCARS, the noise in the probe spectrum is imprinted onto the corresponding spectral regions of the anti-Stokes light. b, Diagrams of various experimental configurations. In (i), the random spectral noise is applied to the probe pulse by the spatial light modulator (SLM) in the pulse-shaper configuration. In (ii), the phase of the probe is randomized owing to the intermodal dispersion of a multi-mode optical fibre. In both cases, the pump and Stokes pulses are generated by the optical parametric amplifier (OPA). In the single-pulse configuration (iii), a diffuser is introduced inside the compressor stage (C) of the regenerative amplifier (RGA) system. Random scattering results in the partial decoherence of the pulse required by NASCARS. The anti-Stokes spectrum is detected by the CCD-based spectrometer, and the autocorrelation is calculated by the computer. LWP: long-wave pass filter; SWP: short-wave pass filter.

of a broad spectrum advances the method of noise-assisted spectroscopy towards efficient scan-less single-beam frequencyresolved analysis of molecular vibrations.

Consider the scheme shown in Fig. 1a with two Raman modes at frequencies $\Omega_{1}$ and $\Omega_{2}$ excited by the pump-Stokes pulse pair. The anti-Stokes field $E(t)$, generated by the Raman scattering of the probe field $E_{0}(t)$, can be expressed as the real part of $E(t)=E_{0}(t) \cdot \mathrm{e}^{-\gamma t}\left[\mathrm{e}^{-i \Omega_{1} t}+\mathrm{e}^{-i \Omega_{2} t}\right]$, where for the sake of clarity we have neglected the non-resonant background, and assumed equal strength and decay rate $\gamma$ for both vibrational modes. The experimentally observed anti-Stokes spectrum $I(\omega)$ is the Fourier transform of the field correlation function $G_{E}(\tau)$, where

$$
G_{E}(\tau)=\int \mathrm{d} t E(t) E^{*}(t-\tau)=G_{\mathcal{E}_{0}}(\tau)\left[\mathrm{e}^{i \Omega_{1} \tau}+\mathrm{e}^{i \Omega_{2} \tau}\right] .
$$

Here, $G_{\mathcal{E}_{0}}(\tau)$ is the correlation function of the modified input probe field $\mathcal{E}_{0} \equiv E_{0} \cdot \mathrm{e}^{-\gamma t}$, that is, the probe field multiplied by the exponential decay of the vibrational coherence. Hence,

$$
\begin{aligned}
I(\omega) & =\int \mathrm{d} \tau \mathrm{e}^{-i \omega \tau} G_{\mathcal{E}_{0}}(\tau)\left[\mathrm{e}^{i \Omega_{1} \tau}+\mathrm{e}^{i \Omega_{2} \tau}\right] \\
& =\mathcal{I}_{0}\left(\omega-\Omega_{1}\right)+\mathcal{I}_{0}\left(\omega-\Omega_{2}\right),
\end{aligned}
$$

where $\mathcal{I}_{0}\left(\omega-\Omega_{1,2}\right)$ is the spectrum of $\mathcal{E}_{0}$. Equation (2) shows that any amplitude noise in $\mathcal{I}_{0}$ is mapped onto the corresponding spectral region of the anti-Stokes spectrum $I$ (that is, up-shifted by the corresponding Raman shift $\Omega_{1,2}$ as schematically shown in Fig. 1a). Averaging over multiple noise realizations, we arrive at the expression for the noise autocorrelation for the measured anti-Stokes spectrum as a function of the frequency shift $\Delta \omega$ :

$$
\left\langle G_{I}(\Delta \omega)\right\rangle=G_{\mathcal{I}_{0}} \cdot[2 g(\Delta \omega)+g(\Delta \omega+\delta \Omega)+g(\Delta \omega-\delta \Omega)],
$$

where $\delta \Omega \equiv \Omega_{2}-\Omega_{1}$ is the distance between the vibrational resonances, and we have assumed an uncorrelated probe noise with $G_{\mathcal{I}_{0}}(\Delta \omega) \equiv G_{\mathcal{I}_{0}} g(\Delta \omega)$ and $g(\Delta \omega)$ being the line-shape function peaked at $\Delta \omega=0$ and of width $\delta \omega_{n}$. The two side bands at $\pm \delta \Omega$ in equation (3) illustrate the ability of NASCARS to retrieve the vibrational beating frequency from the frequency correlations in the noisy spectrum of the Raman-scattered light, with the resolution determined by the noise correlation length $\delta \omega_{n}$.

The required amplitude noise in the spectrum of $\mathcal{E}_{0}$ can be introduced by applying either amplitude or phase noise to the input probe field $E_{0}$. Numerical simulation of the NASCARS measurement with a phase-randomized probe field is shown in Fig. 2. In the time domain, the spectral noise breaks the initial short probe pulse into a long and random sequence of smaller pulsesan 'incoherent pulse train' (see Fig. 2b). In the presence of a longlived vibrational coherence, this probe train generates a train of the corresponding anti-Stokes pulses. The anti-Stokes train is an exact replica of the original random sequence of the probe pulses with an extra periodic amplitude modulation superimposed on it by the vibrational field of the molecule. It is this amplitude modulation that introduces correlations in the otherwise uncorrelated noise in the observed anti-Stokes spectrum, shown in Fig. 2c. High spectral 

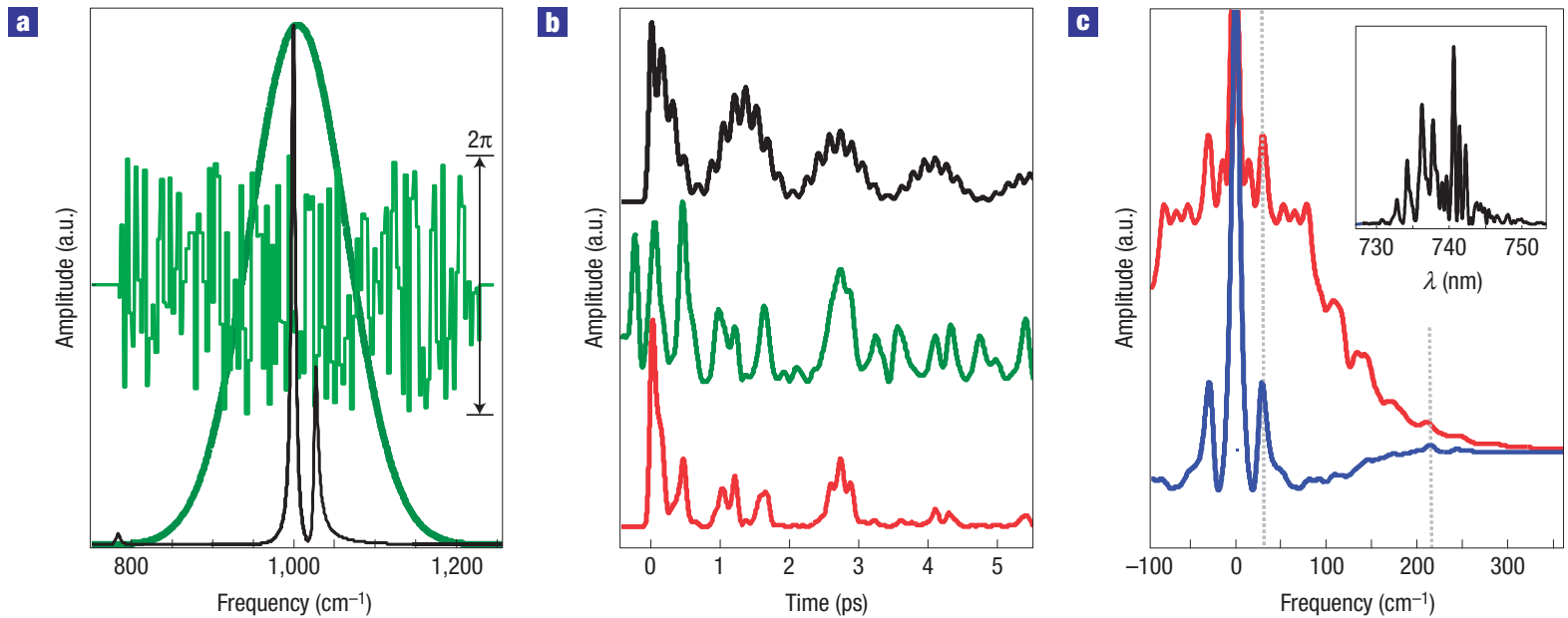

Figure 2 Numerical simulations of the noise autocorrelation spectroscopy with CARS. a, The frequency bandwidth of the probe pulse, represented by the broad gaussian envelope, is much broader than the width of and the distance between the vibrational resonances of toluene (black curve). White noise is applied to the spectral phase of the probe pulse via random phase jumps of $0-2 \pi$ radians every $1 \mathrm{~cm}^{-1}$. b. Time-domain representation of the decaying molecular vibrations (black line), the incoherent probe pulse train obtained by applying the spectral phase noise to the broadband probe (green line) and the resulting anti-Stokes pulse train (red line). Being a product of the vibrational amplitude and the probe field amplitude, the output anti-Stokes field acquires spectral correlations owing to the periodic vibrational modulation superimposed onto the uncorrelated input noise. c, Autocorrelation of the calculated noisy anti-Stokes spectrum (shown in the inset, and exhibiting no clear evidence of the Raman modes) with a single realization of the phase noise (red line) and after averaging over 100 noise realizations (blue line). The strong peak at $27 \mathrm{~cm}^{-1}$ and weak peak at $218 \mathrm{~cm}^{-1}$, marked by the dotted lines, correspond to the beating of the vibrational modes of toluene at $782,1,000$ and $1,027 \mathrm{~cm}^{-1}$.

resolution is achieved when the probe train duration is comparable to the decay time of the vibrational coherence.

We first demonstrate the new method by implementing it in a typical multi-colour non-collinear BOXCARS geometry ${ }^{1}$ (see Fig. $1 \mathrm{~b}(\mathrm{i})$ ). The set-up consisted of a commercial Ti:sapphire regenerative amplifier providing the probe pulses at a central wavelength of $800 \mathrm{~nm}$, and an optical parametric amplifier generating the pump and Stokes pulses at 1,111 and $1,250 \mathrm{~nm}$, respectively. All three pulses were focused into a quartz cuvette containing a liquid mixture of toluene with orthoxylene (see the Methods section for details). The anti-Stokes spectrum was measured with a CCD (charge-coupled device)based spectrometer, whereas the noise was introduced by means of a home-made programmable spectral pulse shaper. The autocorrelation spectrum shown in Fig. 3a was averaged over 100 realizations of noise. Well-resolved side bands at 27 and $49 \mathrm{~cm}^{-1}$ in Fig. 3a correspond to the beating frequencies between the strongest excited vibrational modes of the mixed sample. In agreement with our theoretical treatment and numerical simulations, the best performance of NASCARS is obtained with a maximum $(2 \pi$ peakto-peak) phase modulation, whereas the spectral resolution can be controlled through the pulse shaper by varying the distance between the consecutive phase jumps in the pulse spectrum, $\delta \omega_{n}$. An easier way of scrambling the probe phase without the shaper is shown in Fig. 1b(ii). When coupled to a standard multimode fibre, the probe pulse breaks apart into an incoherent pulse train owing to the intermodal dispersion, and generates similar autocorrelation spectra.

Numerous applications in vibrational spectroscopy, microscopy and imaging call for a single-pulse technique, recently developed by many groups ${ }^{9,10,24,25}$ and based on covering the pump, Stokes and probe wavelengths with a broad spectrum of a single ultrashort pulse. Here, we demonstrate a single-pulse NASCARS measurement, based on the following mechanism. If the applied noise introduces only partial decoherence of the original pulse, the remaining coherent part executes an impulsive pump-Stokes excitation, whereas the incoherent noisy part of the pulse serves as a probe. To NASCARS' advantage, such partial decoherence can be imposed on a laser beam without an elaborate shaping technology, which typically requires delicate optical alignment, calibration, maintenance and costly optical components. We implemented a single-pulse NASCARS by simply introducing a light scatterer inside the pulse compressor of our laser system (see Fig. 1b(iii) and the Methods section for details). Placed in the way of a spectrally dispersed beam, the scattering element-a single sheet of a standard lens paper-transmits only some frequency components of the pulse while blocking the others. Standard pulse characterization shows that in accord with the aforementioned requirement, the output laser pulse consists of an ultrashort coherent part and a long noisy tail. Statistical averaging over the realizations of noise is carried out by moving the scatterer between the consecutive readouts of the anti-Stokes spectrum. The resulting autocorrelation spectrum of $\mathrm{CBrCl}_{3}$, shown in Fig. 3b, exhibits three clearly resolved peaks at the expected beating frequencies of the molecule, attesting to the feasibility of the proposed method. The whole set-up consists of a single slightly modified light source and a spectrometer, yet the achieved resolution of $20 \mathrm{~cm}^{-1}$ surpasses the spectral bandwidth of our pulses $\left(>500 \mathrm{~cm}^{-1}\right)$ by more than an order of magnitude.

The demonstrated implementation simplicity of noise autocorrelation spectroscopy with broadband pulses positions it as a powerful tool for chemical analysis. In all practical cases, the retrieved autocorrelation of the vibrational spectrum retains the uniqueness of the spectrum itself, and can therefore be used as an excellent fingerprint for molecular identification. As the average difference between the beating frequencies is usually smaller than the average frequency of vibration, the resolving power of NASCARS is lower than that of traditional CARS and may limit its application to relatively simple molecular systems. With no stringent requirements on the quality of the excitation 

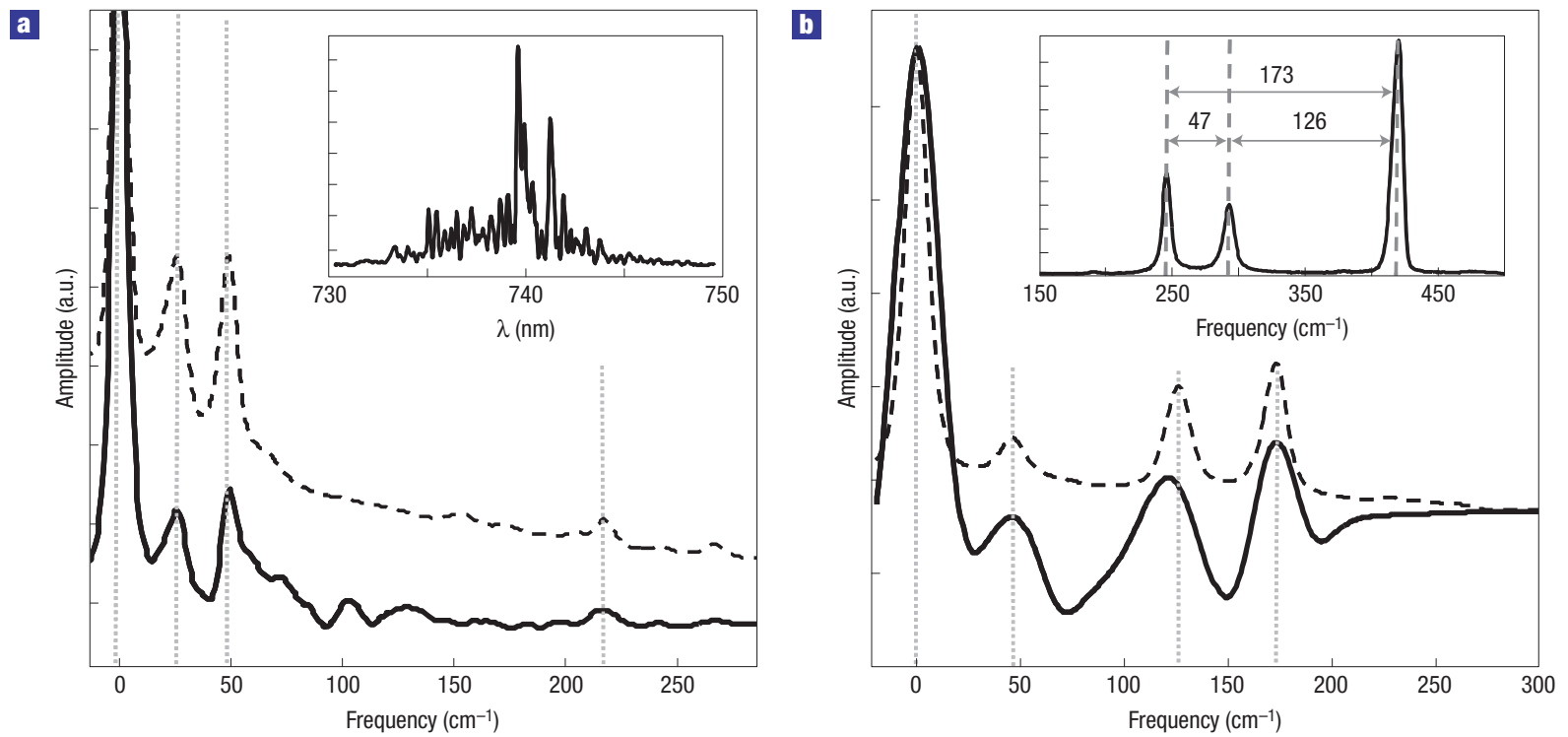

\begin{abstract}
Figure 3 Experimental NASCARS spectra (solid lines) compared with the autocorrelation of the spontaneous Raman spectra obtained by the commercial Raman spectrometer (dashed lines). a, Mixture of toluene and ortho-xylene with the relevant Raman shifts of $782,1,000,1,027,982$ and $1,049 \mathrm{~cm}^{-1}$. Dotted lines point to the beating signals at 27,49 and $218 \mathrm{~cm}^{-1}$. The uncorrelated spectral phase noise was introduced to the probe field through the pulse shaper (Fig. $1 \mathrm{~b}(\mathrm{i})$ ). The anti-Stokes spectrum for a single noise realization is shown in the inset. The observed linewidth is determined by the convolution of the real resonance width of $\sim 3 \mathrm{~cm}^{-1}$ and the spectral resolution of the pulse shaper $\left(\sim 3.5 \mathrm{~cm}^{-1}\right)$ which determines the correlation length of the applied spectral noise. $\mathbf{b}$, Single-pulse NASCARS results for the sample of liquid $\mathrm{CBrCl}_{3}$. Here, a single ultrashort pulse was focused directly in the medium. A thin sheet of a scattering material was placed inside the compressor stage of the laser system (Fig. $1 \mathrm{~b}$ (iii)) to create partial decoherence of the beam. Vibrational beating at 47,126 and $173 \mathrm{~cm}^{-1}$ is clearly identifiable at the expected locations (dotted lines). The observed peaks are associated with the three Raman modes of $\mathrm{CBrCl}_{3}$ at 246, 293 and $419 \mathrm{~cm}^{-1}$, shown on the spontaneous Raman spectrum in the inset.
\end{abstract}

pulse(s), the method may prove especially valuable for applications in nonlinear microscopy by enabling the delivery of the pulses through the standard high-core-area multi-mode optical fibre, normally prohibited because of the intermodal fibre dispersion. In contrast to multiplex CARS, the pump, Stokes and probe pulses in NASCARS are all spectrally broad. As a result, the distribution of the available laser power between these components is independent of the desired spectral resolution and can therefore be adjusted for better performance, for example, by varying the applied noise level. By the very nature of random noise, the required averaging can be carried out in a quick and robust way. Using pseudo-random phase masks, specially designed for the low correlation background, may further reduce the required amount of averaging. In principle, simultaneous detection of multiple anti-Stokes spectra, observed at different scattering angles and, therefore, corresponding to different noise realizations, should enable a single-shot NASCARS measurement.

\section{METHODS}

The experiments in the multi-colour non-collinear geometry (see Fig. 1b(i,ii)) were carried out in a typical CARS set-up, which consists of a commercial Ti:sapphire regenerative amplifier (Spitfire, Spectra-Physics, $1 \mathrm{kHz}$ repetition rate, $10 \mathrm{~nm}$ spectral bandwidth (full-width at half-maximum), $2 \mathrm{~mJ}$ output pulse power) generating the probe pulses at the central wavelength of $800 \mathrm{~nm}$, and an optical parametric amplifier (TOPAS, Light Conversion) generating the pump and Stokes pulses at 1,111 and 1,250 nm, respectively. All three pulses of $\sim 10 \mu \mathrm{J}$ energy each were focused by a lens (focal length of $500 \mathrm{~mm}$ ) into a quartz cuvette ( $5 \mathrm{~mm}$ optical path) with the liquid sample, where they overlapped in time and in space (BOXCARS phase-matching geometry). The output anti-Stokes beam was collimated with a 150 -mm-focal-length lens and coupled into the spectrometer (model 2035, McPhearson) equipped with a CCD camera (iDus DV400, Andor) and providing a spectral resolution of $<2 \mathrm{~cm}^{-1}$.
In the first configuration (see Fig. $1 \mathrm{~b}(\mathrm{i})$ ), we introduced white noise in the probe pulses using the programmable spectral pulse shaper based on a dual-mask 128-pixel liquid-crystal spatial light modulator (SLM-128, CRI). The resolving power of the shaper was $\sim 3.5 \mathrm{~cm}^{-1}$ per pixel, which dictated the smallest frequency correlation length of the applied noise, and therefore determined the spectral resolution of the NASCARS signal. From studying the effects of the phase and amplitude noise independently, we conclude that the phase noise results in superior NASCARS performance. In contrast to the amplitude noise, it does not attenuate the available probe pulse energy and results in a much smaller temporal overlap of the probe train with the pump-Stokes pulse pair, reducing the non-resonant background and increasing the signal quality.

We also demonstrated one simple way of phase scrambling by replacing the pulse shaper with a 3-m-long piece of a standard multi-mode fibre of $64 \mu \mathrm{m}$ core diameter (see Fig. 1b(ii)). A probe pulse, coupled into the multiple fibre modes, breaks apart into an incoherent pulse train owing to the intermodal dispersion. The large number of the available modes and the high sensitivity of the dispersion of each mode to local mechanical stresses in the fibre, enables an easy and efficient way of statistical averaging over the realizations of noise. New realizations of a random probe train are generated by simply moving the fibre between the consecutive data acquisitions. Matching the temporal duration of the output random train to the decay time of the vibrational coherence by choosing an optimal fibre length assures high spectral resolution of the NASCARS measurement.

For the single-pulse experiments, we used ultrashort pulses of higher bandwidth from a different femtosecond Ti:sapphire regenerative amplifier system (Spitfire Pro, Spectra-Physics, $1 \mathrm{kHz}$ repetition rate, $35 \mathrm{~nm}$ spectral bandwidth (full-width at half-maximum), $3 \mathrm{~mJ}$ output pulse power). The wavelength of the amplified pulses was centred at $805 \mathrm{~nm}$. The beam was reduced through an aperture to the diameter of $0.5 \mathrm{~mm}$ before entering the compressor stage of Spitfire Pro. A thin sheet of a light-scattering material (optical cleaning tissue, Thorlabs) was fixed on a rigid frame and mounted on a one-dimensional translation stage inside the compressor (Fig. 1b(iii)). The scatterer was placed after the second reflection from the diffraction grating, that is, at the location where all spectral components of the pulse are parallel and spatially separated. Different noise realizations in this single-pulse configuration 
were achieved by moving the scattering sheet perpendicular to the dispersed beam. The energy of the output beam was $10 \mu \mathrm{J}$. A long-wave pass filter (LP02-785RU-25, Semrock, cutoff wavelength of $785 \mathrm{~nm}$ ) was used to block the high-frequency components of the beam in front of the sample. The laser beam was focused into a quartz cuvette with a liquid sample ( $5 \mathrm{~mm}$ optical path) by a spherical mirror with $300 \mathrm{~mm}$ focal length. The transmitted laser beam was collimated with a lens $(150 \mathrm{~mm}$ focal length) and, after passing a short-wave pass filter (SP01-785RU-25, Semrock, cutoff wavelength of $785 \mathrm{~nm}$ ), coupled into the spectrometer (model 2035, McPhearson) equipped with a CCD camera (iDus DV400, Andor). The resolving power of the spectrometer in this case was $1.3 \mathrm{~cm}^{-1}$.

In all of our experiments, each measured anti-Stokes spectrum corresponded to a different noise realization. After normalization, each two consecutive spectra were subtracted from each other to remove the non-resonant background. An autocorrelation of the differential spectrum represented the result of a single NASCARS measurement. The statistical averaging of the autocorrelation was carried out over 100 noise realizations.

Received 19 July 2007; accepted 2 November 2007; published 9 December 2007.

\section{References}

1. Zheltikov, A. M. Coherent anti-Stokes Raman scattering: From proof-of-the-principle experiments to femtosecond CARS and higher order wave-mixing generalizations. J. Raman Spectrosc. 31, 653-667 (2000)

2. Cheng, J. X. \& Xie, X. S. Coherent anti-Stokes Raman scattering microscopy: Instrumentation, theory, and applications. J. Phys. Chem. B 108, 827-840 (2004).

3. Scully, M. O. et al. Inaugural article: FAST CARS: Engineering a laser spectroscopic technique for rapid identification of bacterial spores. Proc. Natl Acad. Sci. 99, 10994-11001 (2002).

4. Ooi, C. H. R. et al. Theory of femtosecond coherent anti-Stokes Raman backscattering enhanced by quantum coherence for standoff detection of bacterial spores. Phys. Rev. A 72, 023807 (2005).

5. Cheng, J. X., Volkmer, A., Book, L. D. \& Xie, X. S. Multiplex coherent anti-Stokes Raman scattering microspectroscopy and study of lipid vesicles. J. Phys. Chem. B 106, 8493-8498 (2002).

6. Schmitt, M., Knopp, G., Materny, A. \& Kiefer, W. F. Femtosecond time-resolved coherent anti-Stokes Raman scattering for the simultaneous study of ultrafast ground and excited state dynamics: Iodine vapour. Chem. Phys. Lett. 270, 9-15 (1997).

7. Lang, T., Kompa, K. L. \& Motzkus, M. Femtosecond CARS on $\mathrm{H}_{2}$. Chem. Phys. Lett. 310, 65-72 (1999).
8. Oron, D., Dudovich, N. \& Silberberg, Y. Single-pulse phase-contrast nonlinear Raman spectroscopy. Phys. Rev. Lett. 89, 273001 (2002).

9. Lim, S.-H., Caster, A. G. \& Leone, S. R. Single-pulse phase-control interferometric coherent anti-Stokes Raman scattering spectroscopy. Phys. Rev. A 72, 041803 (2005).

10. Dudovich, N., Oron, D. \& Silberberg, Y. Single-pulse coherently controlled nonlinear Raman spectroscopy and microscopy. Nature 418, 512-514 (2002).

11. Pestov, D. et al. Optimizing the laser-pulse configuration for coherent Raman spectroscopy. Science 316, 265-268 (2007).

12. Oron, D., Dudovich, N., Yelin, D. \& Silberberg, Y. Phys. Rev. Lett. 88, 063004 (2002).

13. Xu, X. G., Konorov, S. O., Zhdanovich, S., Hepburn, J. W. \& Milner, V. Complete characterization of molecular vibration using frequency resolved gating. J. Chem. Phys. 126, 091102 (2007).

14. Weiner, A. M. Femtosecond pulse shaping using spatial light modulators. Rev. Sci. Instrum. 71 1929-1960 (2000).

15. Shapiro, M. \& Brumer, P. Principles of the Quantum Control of Molecular Processes (Wiley, New York, 2003).

16. Meshulach, D. \& Silberberg, Y. Coherent quantum control of two-photon transitions by a femtosecond laser pulse. Nature 396, 239-242 (1998).

17. Lu, H. P. \& Xie, X. S. Single-molecule spectral fluctuations at room temperature. Nature 385 143-146 (1997).

18. Schrof, W., Klingler, J. F., Rozouvan, S. \& Horn, D. Raman correlation spectroscopy: A method for studying chemical composition and dynamics of disperse systems. Phys. Rev. E 57, R2523-R2526 (1998)

19. Rom, T. et al. Free fermion antibunching in a degenerate atomic Fermi gas released from an optical lattice. Nature 444, 733-736 (2006)

20. Stimson, M. J., Ulness, D. J. \& Albrecht, A. C. Frequency and time resolved coherent Stokes Raman scattering in CS, using incoherent light. Chem. Phys. Lett. 263, 185-190 (1996).

21. Stimson, M. J., Ulness, D. J. \& Albrecht, A. C. Time-resolved coherent Raman spectroscopy controlled by spectrally tailored noisy light. J. Raman Spectrosc. 28, 579-587 (1997).

22. Kinrot, O., Averbukh, I. S. \& Prior, Y. Measuring coherence while observing noise. Phys. Rev. Lett. 75, 3822-3825 (1995).

23. Averbukh, I. S., Shapiro, M., Leichtle, C. \& Schleich, W. P. Reconstructing wave packets by quantum-state holography. Phys. Rev. A 59, 2163-2173 (1999).

24. von Vacano, B., Buckup, T. \& Motzkus, M. Highly sensitive single-beam heterodyne coherent anti-Stokes Raman scattering. Opt. Lett. 31, 2495-2497 (2006).

25. Paskover, Y., Averbukh, I. S. \& Prior, Y. Single-shot two dimensional time resolved coherent anti-Stokes Raman scattering. Opt. Express 15, 1700-1705 (2007).

\section{Acknowledgements}

The authors are grateful to M. Shapiro and E. Shapiro for helpful discussions. This work has been supported by the CFI, BCKDF and NSERC.

Correspondence and requests for materials should be addressed to V.M.

Reprints and permission information is available online at http://npg.nature.com/reprintsandpermissions/ 